\title{
ON THE GENERAL THEORY OF TRANSFINITE NUMBERS AND ORDER TYPES
}

\author{
By E. W. HoBson.
}

[Received January 26th, 1905.-Read February 9th, 1905.]

1. In accordance with Cantor's general theory of ordinal numbers and of aleph-numbers, there exist two aggregates

$$
\begin{gathered}
1,2,3, \ldots, n, \ldots, \omega, \omega+1, \ldots, \Omega, \Omega+1, \ldots, \beta, \ldots, \\
N_{0}, N_{1}, N_{2}, \ldots, N_{n}, \ldots, N_{\omega}, N_{\omega+1}, \ldots, N_{\Omega}, N_{\Omega+1}, \ldots, N_{\beta}, \ldots ;
\end{gathered}
$$

the first is the aggregate of all ordinal numbers, and the second is that of all $*$ cardinal numbers. These aggregates are both normally ordered (wohlgeordnet) and are similar ta one another; and they contain respectively every ordinal number, and every cardinal number which belongs to a normally ordered aggregate.

In accordance with the principle which is fundamental in the whole theory, that every normally ordered aggregate has a definite order type, or ordinal number, and also a definite cardinal number, it is seen that the above aggregates have an ordinal number $\gamma$ and a cardinal number $\boldsymbol{N}_{\gamma}$. The ordinal number $\gamma$ must itself occur in the first aggregate, and must therefore be the greatest ordinal number, i.e., the last element of the aggregate ; moreover $N_{y}$ must occur in the second aggregate, and must be the last element of that aggregate. There can, however, be no last ordinal number; for, on the assumption of the existence of $\gamma$, an aggregate of ordinal number $\gamma+1$ can be formed; for example, by placing the first element of either of the above aggregates, after $\gamma$ or $N_{y}$; it can then be shown that there can be no last aleph-number $x_{y}$. We have thus arrived at a contradiction.

Burali Forti, who first pointed out this contradiction,* accounted for it by denying the truth of the theorem, that any two distinct ordinal numbers $a_{1}, a_{2}$ must necessarily satisfy one of the relations $a_{1}>a_{2}, a_{1}<a_{2}$, in accordance with Cantor's definition of the meaning of these relations. finite."

Rend. del Circolo mat. di Palermo, Vol. xI., 1897, “Una Questione sui Numeri Trans- 
However, Cantor's proof* of this theorem does not appear to be capable of refutation; so that the origin of the contradiction cannot be explained in the manner indicated.

B. Russell has suggested that the aggregates of all ordinal numbers and of all aleph-numbers are not normally ordered, and therefore that these aggregates have no ordinal number, and that their cardinal number is consequently not necessarily an aleph-number. He admits, however, that the segments of either aggregate are normally ordered. This explanation is confuted by the argument duet to Jourdain, that, if the above aggregates are not normally ordered, then they must contain parts of type ${ }^{*} \omega$; such a part would then be a part also of a segment of one of the aggregates, and such segment would not be normally ordered.

The contradiction has been explained by Jourdain, by means of the suggestion that there are ordered aggregates which have no order type and no cardinal number, and that the above aggregates belong to such class. To such aggregates he gives the name inconsistent aggregates, in virtue of the fact that of such an aggregate it is impossible to think, without contradiction, as a "collection by the mind of definite, distinct objects to a whole." It appears from a statement made by Jourdain, $\downarrow$ that Cantor had himself, some years previously, arrived at the same conception and name. In accordance with this view of the matter there exists an ordered aggregate, that if all the ordinal numbers, every segment of which is normally ordered a id has a cardinal number, and yet the aggregate itself being "inconsistent" cannot, without contradiction, be thought of as having an order type. This amounts to a denial of the universal validity of the fundamental principle that every ordered aggregate has a definite order type ; and yet it is by means of this very principle that the existence of the successive ordinal numbers is regarded as having been established. Each successive ordinal number was defined to be the order type of the ordered aggregate of all the preceding ordinal numbers.

The doubt thus thrown upon the validity of the principle by means of which the existence of the complete series of ordinal numbers, and, simultaneously, that of the aleph-numbers, is established in Cantor's theory, naturally suggests that a further scrutiny of the foundations of that theory is needed. It is not clear, a priori, that an argregate which

* Math. Annalen, Vol, xLrx., p. 215.

† Mhil. Mag., 1904, "On the Transfinite Numbers of well-ordered Aggregates."

$\ddagger$ Loc. cit., p. 67, note. See also Hilbert, Jahresbericht der Deutsch. MIath. Vereing., Vol. virr. p. 184 . 
is inconsistent, in the sense employed above, may not be reached at an earlier stage of the process of forming the successive classes of ordinal numbers, before the aggregate of all such numbers, in the sense of Cantor's theory, is reached. Moreover, it would seem reasonable to expect that so fundamental a distinction, as that involved in the notion of an inconsistent aggregate, should be indicated in the general definition of an ordered aggregate, or in close connection therewith. In any case, an explanation of the contradiction on these lines cannot be regarded as satisfactory, until criteria have been obtained which shall suffice to decide, in respect of any particular ordered aggregate, whether such aggregate has an order type and a cardinal number, or whether it is an inconsistent aggregate.

2. Before proceeding to attempt the consideration of the question how far Cantor's general theory of ordinal numbers and aleph-numbers can be accepted as sound, some remarks will be made as to the definition of an aggregate in general, with a view to discover whether it has; in the form given by Cantor, the requisite degree of precision. An attempt will then be made to decide what limitations or qualifications must be imposed upon the nature of an aggregate, so that in the development of the theory, the possibility of being confronted by such a contradiction as that which was pointed out by Burali Forti may be removed at its source.

Cantor has given the following definition of an aggregate (Menge) :"Menge ist jede Zusammenfassung $M$ von bestimmten wohlunterschiedenen Objecten $m$ zu einem Ganzen, $M=\{m\}$." The term aggregate being thus taken as denoting a collection of distinct objects, in the most general sense, the difficult question arises as to when the elements of the aggregate can be regarded as adequately defined. In the case of a finite aggregate, the elements may be defined by means of individual specification, but this is not possible in the case of a transfinite aggregate. Individual specification must then, in the latter case, be replaced by a law or a set of laws, forming the norm by which the aggregate is defined. The most general definition which presents itself, as a prima facie definition of an aggregate, is that an aggregate consists of all objects each of which satisfies certain specified conditions. It is, however, convenient to admit the case of two or more alternative sets of conditions; thus an aggregate may contain all objects each of which satisfies either the conditions $A$ or else one of the sets of conditions $B, C, \ldots, K$. The conditions forming the norm by which the aggregate is defined must be of a sufficiently precise character to make it logically determinate as regards uny particular object whatever, whether such object does, or does not, 
belong to the aggregate. In such a case as the aggregate of prime numbers, we may practically be unable to decide, as regards a particular number, whether that number is a prime or not, but we know that the question could be settled by means of a process containing a finite number of steps. On the other hand, if we consider the aggregate of all algebraical numbers, we may possess no present means of deciding, by means of a known process, whether a particular number is an algebraical number or not. It would, however, be an undue restriction of the conception of an aggregate, to insist upon the possibility of actual determination by means of a known finite process, whether a particular object satisfies the norm by which the aggregate is defined or not. We therefore agree to fall back upon logical determinacy as sufficient; thus, for example, it is logically determinate as regards a number defined in any particular manner, whether that number is algebraical or transcendental, although we possess no perfectly general method of making the actual determination.

We shall accordingly define the meaning to be attached to the term aggregate as follows:-

All objects which are such as to satisfy a prescribed norm are said to belong to an aggregate defined by that norm. The norm consists of a set of specified conditions, or of a specified set of alternative specified. conditions, and must be sufficient to render it logically determinate, as regards any particular object ,hatever, whether that object belongs to the aggregate or not.

In the case of a finite aggregate, the norm may take the form of individual specification of the objects which form the aggregate.

3. It is not clear that an aggregate, defined in the above sense, is necessarily capable of being ordered at all. For example, it is difficult to see that such an aggregate as that of "all propositions " could conceivably be ordered ; it being assumed that the meaning of the word "proposition" is taken as so definite that this aggregate has a norm, in accordance with the definition above. Again, to take an example among aggregates of the kind usually considered in mathematical theory, we may consider the aggregate obtained by covering (Belegung) the aggregate of real numbers by itself ; this aggregate, which, according to Cantor, has the cardinal number $f \equiv c^{c}$, is equivalent to the aggregate of all functions of a real variable: it is difficult, if not impossible, to see how order could be imposed upon this aggregate. If, then, a transfinite aggregate is to be given as an ordered aggregate, or is to have an order imposed upon it, it would appear to be necessary that the norm which constitutes the defini- 
tion of the aggregate should be of such a character that a principle of order is contained therein, or can at all events be adjoined thereto; so that, when any two perticular elements are considered, the conditions which they satisfy in virtue of their belonging to the eggregate, when individualized for the particular elements, may be sufficient also to assign relative rank to those elements in accordance with a principle of order. This is, in fact, the case in such aggregates as those of the integral numbers, the rational numbers, or the real numbers. In the case, for example, of the positive rational numbers, defined in accordance with the arithmetic theory as associations of pairs of integers, the relative rank of any two particular elements $(p, q),\left(p^{\prime}, q^{\prime}\right)$ is assigned by the system of postulations which defines the aggregate. It may, of course, also be possible in other cases, as in this one, to re-order the aggregate in accordance with some other law extrinsically imposed upon the aggregate; but the nature of the elements must be such that this is possible.

We can now state that:-

In order that a transfinite aggregate, defined as in $\S 2$, may be capable of being ordered, a principle of order must be explicitly or implicitly contained in the norm by which the aggregate is defined.

In the definition of the order type of an aggregate, given* by Cantor, according to which the order type is obtained by making abstraction of the particular nature of the elements of the aggregate, the conception of their order being retained, it is assumed that the aggregate is given as an ordered one. Again, Cantor has defined a cardinal number as the notion which remains, when, by help of our mental activity, we make abstraction of the nature of the elements of an aggregate, and of the order in which they are given. Here the question arises whether the definition of cardinal numbers should not also be applicable in the case of aggregates which are not given as ordered aggregates. Cantor has himself, in fact, in his theory of exponentials which involve transfinite numbers, contemplated certain aggregates as having cardinal numbers, whilst such aggregates are not given as ordered aggregates, and, prima facie, at least, are not capable of being ordered.

The relative order of any two elements of a transfinite ordered aggregate is determined by the individual characteristics of those elements (including, when relevant, their positions in space or time), subject to the defining norm. If then, complete abstraction be made of the nature of the elements, they become indistinguishable from one another, and 
order in the aggregate is thereby obliterated; all relation to the norm by which the original aggregate was defined having disappeared.

4. Taking the case of an aggregate defined as an ordered aggregate, we now approach the consideration of the fundamental question whether, and under what conditions, if any, such an aggregate can be regarded as having a definite order type and a definite cardinal number. This is equivalent to asking whether, or when, meaning can be given to these terms, of such a character that they can be treated as denoting permanent objects for thought, or as mathematical entities which may themselves be elements in aggregates.

There are two distinct methods of establishing* the existence of a class of mathematical entities :-

(1) Their existence, as detinite objects for thought, may be shown to follow as a logical consequence of the existence of other entities already recognized as existing, or of principles already recognized as valid; thus the existence of the new entities in question cannot be denied without coming into contradiction with truths already known. This method may be termed the genetic method.

(2) The existence of the entities may be postulated; their mutual relations and their relations with other entities being defined by means of a complete system of definitions and postulations. Accordingly, the objects in question are a free creation of our mental activity. The validity of the scheme thus set up is established when it is shewn to be free from internal contradiction and from contradiction with other known truths. Its utility is to be judged by its applicability to the general purposes of the science, and the light it may throw upon the fundamental principles of that science, in virtue of the scheme containing a generalization of what was previously known. This method may be termed the method of postulation.

Both these methods have been employed by Cantor in his theory of transfinite numbers and order types. In his earlier treatment of the subject he employed the second of the above methods. The existence of the new number $\omega$, and of the other limiting numbers of the second class, was postulated in accordance with his second principle of generation (Erzeugungsprinzip). Freedom from contradiction, and utility in connection with the theory of sets of points, which suggested the postulations, were relied upon as the grounds upon which the scheme of new numbers

* See Hilbert, Jahresbericht d. deutseh. math. Vereing., Vol. virr., p. 180. 
was to be justified. The first number $\Omega$, of the third class, was introduced by a new postulation.

In his later and more abstract treatment of the subject, Cantor applied the genetic method. The existence of the number $\omega$ is not directly postulated, but is taken to follow from the existence of the aggregate $\{n\}$ of integral numbers; $\omega$ is defined to be the order type of this aggregate, and it is assumed that such order type is a definite object which can itself be an element of an aggregate. The existence, as definite entities, of the cardinal numbers being assumed, the successive ordinal numbers of the successive classes are obtained, by assuming the general principle that an ordered aggregate necessarily possesses a definite order type which can be regarded as an object, viz., the ordinal number coming immediately after all those which are the elements of the aggregate of which it is the order type.

5. It has been seen above that the assumptions that an ordered aggregate necessarily possesses a definite order type and a definite cardinal number, which can be treated as objects, lead to the contradiction pointed out by Burali Forti. It appears, therefore, that the classes of entities, the ordinal numbers of all classes, and the similar aggregate of aleph-numbers, do not satisfy the condition of being subject to a scheme of relations which is free from contradiction. In fact, the principle in accordance with which their existence is inferred is in conflict with the definition of the aggregates, as containing every ordinal number, or every aleph-number. It would then appear that the genetic process, which led to the definition of all ordinal numbers and of all aleph-numbers, cannot be a valid one. Thus, the principle that every ordered aggregate has a definite order type which may be regarded as a permanent object for thought cannot be accepted as a universal principle to be used in a genetic mode of establishment of the existence of a class of entities. A denial of the validity of this principle does not, however, preclude the less ambitious procedure of postulating the existence of definite ordinal numbers of a limited number of classes, in accordance with Cantor's earlier method. So long as the postulation of the existence of ordinal numbers does not go beyond some definite point, no contradiction will arise, and the utility of the scheme, for purposes of representation, will suffice to justify the postulations which have been made. An attempt to examine the structure of such a class of ordinal numbers as that of the $\omega$-th class, with cardinal number $N_{\omega}$, or that of the $\Omega$.th class, with cardinal number $N_{\Omega}$, will lead to the conviction that such conceptions are unlikely to be capable of useful application in any branch of analysis or geometry for purposes of 
representation. Nevertheless, in case inexorable logic compelled us to contemplate the existence of such classes of objects, they would form a very proper field of exploration; we have, however, seen that there are grave doubts as to whether this is the case.

6. The genetic method being rejected on the ground that it leads to the construction of a class of entities which, in its entirety, can have no existence, we have to fall back upon the method of postulation. A consideration of the essential elements in the conceptions which lie at the base of the scheme of finite integral numbers may afford guidance as to how far we may properly proceed in the construction, by postulation, of transfinite ordinal numbers of successive classes. The ordinal numbers of any one particular class are those which belong to rearrangements of the elements of an aggregate of which the order type is the lowest number of that class. We may, therefore, consider primarily the lowest numbers of the classes of which the cardinal numbers are $N_{0}, N_{1}, N_{2}, \ldots$ respectively. A finite ordinal number is characteristic of a class of aggregates all of which are similar to one another, and a finite cardinal number is characteristic of a class of aggregates all of which are equivalent to one another; thus, in both cases, the notion of correspondence between the elements of different aggregates is an essential factor in the idea of number. The number of each of a class of similar or of equivalent aggregates is considered to be definite object. The relation of this object to any member of the cla.s of which it is characteristic may be illustrated by the relation of the colour red to a particular red object.

The existence of a particular number does not follow as a mere logical consequence of the existence of the preceding numbers, but from the existence of the class of aggregates of which it is characteristic, the relation between different aggregates of the class being that of $(1,1)$ correspondence. In the genetic method, as applied to the construction of the whole series of classes of transfinite ordinal numbers, this notion of correspondence plays no part; in fact, the existence of a number is constantly inferred from that of a single unique ordered aggregate. For example, the existence of $\Omega$ and of $N_{1}$ is inferred from the existence of the single aggregate of numbers of the first and second classes. Generally, in the whole scheme, the existence of a new number is inferred from the existence of that unique aggregate which contains the preceding ordinal numbers. That this procedure leads to contradiction has been already seen. The transfinite numbers must be regarded as obtained or defined in accordance with the same principles as obtain in the case of the finite numbers, if they are to be regarded as numbers, even in on extended

BER. 2. vOr. 3. พо. 893. 
sense of the term. It seems, then, highly probable that the neglect of the principle, that correspondence between similar or between equivalent aggregates is essential to our right to consider the numbers belonging to aggregates as definite entities, may be the source of the contradiction which arises from the thorough-going application of the genetic method which leads to Cantor's complete series of ordinal numbers and aleph-numbers. In accordance with this view of the nature of number, finite or transfinite, the postulation of the existence of a definite entity, which entity shall be entitled to be regarded as a number, is only justified when it is shown that other aggregates exist besides the aggregate which consists of the preceding ordinal numbers, of which other aggregates the postulated number is the characteristic number. Thus the postulation of the numbers $\omega$ and $N_{0}$ requires for its justification the exhibition of other aggregates besides $\{n\}$, that of all finite numbers; in this case the requirement is satisfied by the definition of sets of points or of other geometrical objects, and thus there really exists a class of aggregates which are all similar to the ordered aggregate $1,2,3, \ldots, n, \ldots$; and hence the postulated order type $\omega$ and the postulated cardinal number $N_{0}$ are really entitled to rank as ordinal and cardinal numbers respectively. When we consider the ordinal number $\Omega$, and the cardinal number $N_{1}$, the state of the case is very different. In order that the existence of $\Omega$ might be on a parity with that of $\omega$, it would require to be shown that it is possible to define a set of objects, say points of the linear continuum, which should be such that to each prescribed ordinal number of the second class there corresponds a definite point of the continuum, i.e., to show that a norm is possible which would define a set of points of order type $\Omega$. This has hitherto* not been accomplished, nor have aggregates having any of the cardinal numbers $N_{2}, N_{8}, \ldots$ been defined by means of definite sets of rules. If it be urged that the postulation of the existence of the order type $\Omega$, and of the corresponding cardinal number $N_{1}$, does not of itself lead to contradiction, it may be replied that such postulation does not entitle $\Omega$ and $N_{1}$ to rank as numbers in the sense in which $\omega$ and $N_{0}$ are numbers; for in the latter case the essential elements in the original conceptions of ordinal and cardinal numbers are all present, wheress this has not been shewn to be true of $\Omega$ and $N_{1}$. Moreover, the postulation of the existence of $\Omega$ and $N_{1}$, if it does not of itself lead to contradiction, can only be made by means of a principle which, when applied systematically, certainly leade to contradiction. In accordance with the criterion laid down above,

- An attempt to define a set of points of cardinal number $N_{1}$ has been made by $G$. $H$. Hardy ; this will be criticized in $\$ 12$. 
$N_{1}, N_{2}, \ldots$ cannot at the present time be regarded as definite entities, and could not be regarded as in any true sense numbers, even if any meaning could be assigned to them.

It may conceivably turn out, in the future, to be possible to justify the postulation of certain of the numbers $N_{1}, N_{2}, \ldots$ with the classes of ordinal numbers which would belong to them ; it will, however, certainly never be possible to do this for the whole class $\left\{\sim_{\beta}\right\}$, where $\beta$ is any ordinal number of the aggregate of all ordinal numbers, in accordance with Cantor's complete scheme, because such postulation leads to unavoidable contradiction. The setting up of a scale of standards, to which standards no aggregates not consisting of the preceding numbers conform, involving as it does the employment of sphinx-like aggregates, to each of which no other aggregate can be shown to be similar, would, a priori, appear to be an illegitimate extension of the notion of number, from which an essential element had dropped out, and, a posteriori, it has been shown to lead to contradiction.

It may be urged that no contradiction would ensue if, in single instances, the existence of order types and powers, considered as definite entities, were postulated for aggregates of the unique character referred to above. But, if this were done, such order types and powers would not be entitled to rank as numbers, and such sporadic creations would be of no importance in mathematical heory. 'Systematic postulation of this character is just what has bee: shown to lead to a self-contradictory scheme of entities, and is therefore illegitimate.

7. A cardinal number has been defined* by B. Russell to be a class of equivalent aggregates. It may then be urged that such class may contain only one member, and that this is sufficient for the existence of the cardinal number. In fact, Russell infers the existence of the number $n+1$ from the mere existence of the numbers $0,1,2, \ldots, n$. In accordance with the view here advocated, this definition, or any other one which allows the existence of a cardinal number to be inferred solely from the existence of a unique aggregate, to which no other aggregates have been shown to be equivalent, must be rejected. Russell objects $\$$ to the conception of a number as the common characteristic of a family of equivalent aggregates, on the ground that there is no reason to think that such a single entity exists, with which the aggregates have a special relation, but that there may be many such entities. The mind does, however, in point of fact, in the case of finite aggregates at least, recognize the existence of such single

* Principles of Mathenatics, Vol. I., p. 111-116.

$\dagger$ Ibid, p. 497. 
entity, the number of the aggregates; and this is a valid result of our mental activity, subject to the law of contradiction. Since Russell apparently regards the activities of the mind as irrelevant in questions of existence of entities, his view, and the one here advocated, do not rest upon common premisses. An advantage claimed for the view here supported, over that of Russell, is that it does not lead to such a contradiction as that pointed out by Burali Forti.

The definition of cardinal number, by abstraction, which Cantor employs, can be justified only by the interpretation, that abstraction is made only of those characteristics in which the elements of the aggregate differ from the corresponding elements of equivalent aggregates. The partial abstraction will then consist in taking the cardinal number to be the common characteristic of all such equivalent aggregates. To make a complete abstraction of the nature of the elements of a transfinite aggregate would remove the relation with the cardinal number.

8. The conclusions at which we have arrived in the course of the above discussion may now be summarized as follows :-

(1) The aggregates

$$
\begin{array}{r}
1,2,3, \ldots, n, \ldots, \omega, \omega+1, \ldots, \Omega, \ldots, \beta, \ldots ; \\
N_{0}, N_{1}, \ldots, N_{n}, \ldots, N_{\omega}, N_{\omega+1}, \ldots, N_{\Omega}, \ldots, N_{\beta}, \ldots
\end{array}
$$

of all ordinal numbers, and of all aleph-numbers, in the sense in which Cantor contemplates them, have no existence. Their existence cannot be established without the assumption of the principle that every normally ordered aggregate necessarily has a definite order type and a definite cardinal number which can themselves be regarded as objects capable of being elements of an aggregate. This principle leads to contradiction, and must therefore be rejected as not being a universally valid truth.

(2) Of the aleph-numbers, the postulation of the existence of $N_{0}$ has hitherto alone been justified, by showing that it is possible to define aggregates consisting of objects other than the ordinal numbers themselves, of which it is the characteristic cardinal number. The numbers $\omega, \omega+1, \ldots, \omega .2, \ldots, \omega^{2}, \ldots$ of the second class exist, but it has not yet been shewn that the totality of all these numbers, taken in order, has a definite order type, or a cardinal number. To do this it would be necessary to shew that a finite set of rules can be set up which will suffice to define a definite object corresponding to each ordinal number of the second class.

(3) The existence of individual aleph-numbers, other than $\aleph_{0}$, with 
the classes of ordinal numbers belonging to them, may, in the future, possibly be established, but it is not possible that this should be done beyond some definite point.

It may be observed that an aggregate which consists wholly of distinct physical objects which do not penetrate one another, must be enumerable (abzählbar), for each such object occupies some finite volume in space; and it has been shewn by Cantor that any set of non-overlapping portions of space is enumerable. It follows that the objects in an unenumerable aggregate must, with the possible exception of an enumerable component of the aggregate, consist of ideal or abstract objects.

9. The regarding of a collection as a "whole" has been emphasized by Cantor as essential to the notion of an aggregate. It is no doubt true that, in a certain sense, every logical class, or every aggregate as defined in $\S 2$, forms a whole, as being dominated by a norm; but, for the purposes of mathematical science, the important question is under what circumstances such an aggregate may be regarded as having a definite cardinal number, and, if ordered, a definite order type. An attempt has been made above to answer this question in the case of normally ordered aggregates; the answer in the case of other aggregates would be on the same lines.

Ordered aggregrates have bee 1 defined which are not normally ordered ; and of such aggregates the $\mathrm{n}$ ost important is the arithmetic continuum defined as of the order type $\theta$, considered* in detail by Cantor. That $\theta$ is a definite object, with a corresponding definite cardinal number $c$, must, as has already been pointed out, be regarded as a postulation subject to the law of contradiction. It is known that a class of aggregates exists which are similar to the linear continuum, and thus conform to the type $\theta$, and have $c$ as their common cardinal number; this is in accordance with the regulative principle which we have maintained to be essential to justify our regarding $c$ as a number.

As has been already remarked, aggregates may be defined which are unordered. In such cases no question arises as to the existence of an order type, but there is no reason why such aggregates should not have cardinal numbers, provided that in the case of such an aggregate, equivalent aggregates can be found of which the cardinal nuruber in question is the common characteristic. The aggregates of whish the cardinal number is $c^{c} \equiv f$ are an example of this species of aggregate. Two 
aggregates which have been independently defined are not necessarily comparable with one another as regards either order type or cardinal number. It cannot be assumed a priori that the cardinal number of one of them is necessarily either greater than, equal to, or less than that of the other, in the sense in which these relations are defined in the theory. Further, it cannot be assumed that an ordered aggregate such as, for example, the continuum, is necessarily capable of being normally ordered. There is, as we have seen, no convincing reason for thinking that any unenumerable aggregate is capable of being normally ordered. Two aggregates of abstract objects, which have been independently defined, belong, no doubt, to the same universe of thought; but, nevertheless, any particular category of relations may be too narrow to formulate any nexus between the two systems, so that it is conceivable that, so far as such relations as those of order and cardinal number are concerned, the two aggregates may be completely isolated from one another.

10. In some proofs of theorems which have been given by writers on this subject, which proofs have for their object the establishment of relations of inequality or equality of cardinal numbers, aggregates are employed, the elements of which are regarded as being successively defined by an endless series of separate acts of choice. When we leave the region of the finite, it would, however, appear that we have passed beyond the region in which definitions by arbitrary acts of choice can be regarded as adequate specifications of definite objects; the existence of a norm would appear to be essential to our right to regard an aggregate as really defined, and therefore to justify our making use of the conception of such an aggregate in the proof of a theorem. The point may be illustrated by a discussion given* by P. Du Bois Reymond, in which he contemplates the existence of a number represented by a non-terminating decimal in which the figures are determined by no law. He contemplates that each figure in the decimal may be fixed by a throw of dice, and rejects the conception of such a decimal (ewig gesetzloses Decimal) as representing a real number. A non-finite, or endless, process can be conceived of as a completed whole, only when it is subject to some kind of norm; thus a non-terminating decimal represents a number, only under the presupposition that a set of rules can be given which suffice to determine the figure which occupies any assigned place in the decimal. In general, the proof of the possibility of giving 
a norm is required before an aggregate of any particular character can be contemplated as existing, or can be legitimately made use of in a demonstration.

Cantor, in his proof that $\boldsymbol{N}_{0}$ is less than any other transfinite cardinal number, has assumed that it is possible to pick out of any given transfinite aggregate an enumerable component. This proof can only be accepted as valid in case it is possible to detine an enumerable component of the aggregate in question. In a large class of cases-possibly in all which are of importance in mathematical theory-this condition can be satisfied: for uxample, in the case of the continuum. In the aggregate of "all propositions," for example, the enumerable component may be taken to be that aggregate of propositions which asserts the existence of the numbers $1,2,3, \ldots$.

G. H. Hardy has extended * Cantor's method, for the purpose of shewing that every transfinite cardinal number is either an aleph-number, or is greater than all the aleph-numbers; and, in particular, $2^{N_{0}}=c \geqslant N_{1}$. The proof runs as follows:-Having given an aggregate whose cardinal number is $>N_{0}$, we can choose from it successive individuals

$$
u_{1}, u_{2}, \ldots, u_{\infty}, \ldots, u_{\beta}, \ldots
$$

corresponding to all the numbers of the first and second classes; if the process came to an end, 'ae cardinal number would be $N_{0}$. Its cardinal number is therefore $\geqslant N_{1}$; and, if $>N_{1}$, it is $\geqslant N_{2}$; and so on. And, if $>N_{n}$, for all finite values of $n$, it must be $\geqslant N_{a}$; for we can choose individuals from the aggregate corresponding to all the numbers of the first, second, $\ldots, n$-th, $\ldots$ classes. And, by a repetition of these two arguments, we can shew that, if there is no $N_{\beta}$ equal to the cardinal number of the aggregate, it must be at least equal to the cardinal number of the aggregate of the $N_{\beta}$, and so is greater than any $N_{\beta}$.

Apart altogether from the question as to what constitutes all the aleph-numbers, this argument could only be made valid if it were shewn how the successive individuals $u_{1}, u_{2}, \ldots, u_{\beta}, \ldots$ are to be defined by means of some norm, and also how the individuals of the aggregate which may correspond to the numbers of the first, second, $\ldots, n$-th, $\ldots$ classes can be assigned by a norm. The process can neither come to an end, nor be regarded as, in any sense, a completed one, unless this has been done. 
11. Two proofs have been advanced, that every cardinal number is necessarily an aleph-number; this is equivalent to the statement that every aggregate which has a cardinal number can be normally ordered. If these proofs could be accepted as valid, the particular theorem would be established, that the arithmetic continuum is capable of being normally ordered; and the only question which would remain open, as regards this aggregate, would be as to which particular aleph-number is the cardinal number of the continuum.

The first of these proofs, that of Jourdain, ${ }^{*}$ is founded on the assumption that, if a cardinal number is greater than every aleph, there must be a part of the aggregate to which this cardinal number belongs which can be made to have a $(1,1)$ correspondence with the "inconsistent" aggregate of all the ordinal numbers arranged in normal order. This assumption is regarded as justified by the process of making the successive elements of the aggregate of ordinal numbers correspond to elements of the given aggregate; it is then argued that, if this process comes to an end, the cardinal number of the aggregate is an aleph, and, if it does not come to an end, the given aggregate must contain as a part the "inconsistent" aggregate of all the ordinal numbers; thus in the latter case the aggregate is inconsistent, and has no cardinal number.

The objection to this proof is of the nature which hai been already stated, viz., that no norm is forthcoming by which the correspondence in question is defined; and, in default of such norm, there is no meaning in speaking of an essentially endless process as a completed one, or as having come to an end.

In the second proof, duet to E. Zermelo, no account is taken of the possibility that an aggregate may have no cardinal number, or of the existence of "inconsistent" aggregates. The proof, which is fundamentally of a similar character to that of Jourdain, is represented as demonstrating that every aggregate can be normally ordered, and thus has an aleph as its cardinal number.

It is assumed that in each part $M^{\prime}$ of a given aggregate $M$, one element $m^{\prime}$, called the special (ausgezeichnetes) element of $M^{\prime}$, can be chosen. A part $M^{\prime}$ must contain one element of $M$ at least, and may contain all the elements; and the aggregate $\left\{M^{\prime}\right\}$ of all parts of $M$ is considered. Each element $\boldsymbol{M}^{\prime}$ of $\left\{\boldsymbol{M}^{\prime}\right\}$, corresponds to a special element which kzlongs to $M^{\prime}$, and this particular mode of covering the elements of $\left\{M^{\prime}\right\}$ by elements of $M$, is called a " covering " $\gamma$; the employment of a particular "covering" $\gamma$ is essential to the proof. A $\gamma$-aggregate

- Phil. Mag., January, 1904, pp. 67, 70.

+ Math. Annalen, Vol. LIX, 1904, "Beweis, dasz jede Menge wohlgeordnet werden kann." 
is then defined as follows :- Let $M_{\gamma}$ be a normally ordered aggregate consisting of different elements of $M$, such that, if $a$ is any arbitrarily chosen element of $M_{\gamma}$, and $A$ is the segment ( $A$ bschnitt) of $M_{\gamma}$ defined by $a$, which segment consists of all the elements of $M_{\gamma}$ which precede $A$, then $a$ is always the special element of $M-A$. Every such aggregate $M_{\gamma}$ is a $\gamma$-aggregate. If every element of $M$ which occurs in a $\gamma$-aggregate be called a $\gamma$-element of $M$, it is shewn that the aggregate $L_{\gamma}$ of all $\gamma$-elements can be so ordered that it is itself a $\gamma$-aggregate, and contains all the elements of the original aggregate $M$. It follows then that $M$ can be normally ordered.

Zermelo himself expressly recognizes the assumption made as to the existence of a definite "covering" $\gamma$. The objection to this assumption is of the same character as before, viz., that for its validity a norm must be shewn to be possible; this norm must assign to each part of the given aggregate, a definite " special " element belonging to that part. In the case of such an aggregate as the arithmetic continuum, it is not clear how such a norm could be devised; indeed, it seems probable that a proof of the possibility of establishing such a norm involves difficulties comparable with those which occur in any attempt to prove the original theorem. The non-recognition of the existence of "inconsistent" aggregates, which existence, on the assumption of Cantor's theory, cannot be denied, introduces an additional eleme t. of doubt as regards this proof. The aggregate $L_{\gamma}$ here employed is parallel with the normally ordered aggregate which occurs in Jourdain's earlier proof.

12. [Added February 25th, 1905.] - A method has been given* by G. H. Hardy for the construction of a set of points of cardinal number $\aleph_{1}$. $\mathrm{He}$ proposes to determine for each ordinal number $\beta$ of the first or of the second class, a sequence $b_{1}, b_{2}, b_{8}, \ldots, b_{n}, \ldots$, consisting of increasing integers, so that to each number $\beta$ a unique sequence corresponds which is not identical with the sequence which corresponds with any other ordinal number of the first two classes. The sequence is then correlated with a point of the linear continuum $(0,1)$, by taking that binary radix fraction in which the $b_{1}$-th, $b_{2}$-th, $b_{3}$-th, ... figures are all 1 , whilst the remaining figures are all 0 ; the point of the linear continuum so defined being taken to correspond to $\beta$. The sequence corresponding to the number 1 being taken to be $1,2,3,4,5, \ldots$, the othr.f sequences are determined by the following modes of formation :-

(1) If $b_{1}, b_{2}, b_{9}, \ldots, b_{n}, \ldots$ corresponds to $\beta$, then $b_{2}, b_{3}, b_{4}, \ldots, b_{n+1}, \ldots$ is the sequence which corresponds to $\beta+1$. 
(2) The sequence which corresponds to a limiting number $\gamma$ of the second class is obtained from the sequences

$$
\begin{array}{llll}
a_{11}, & a_{12}, & a_{13}, \ldots, & a_{1 n}, \ldots, \\
a_{21}, & a_{22}, & a_{23}, \ldots, & a_{2 n}, \ldots, \\
a_{31}, & a_{92}, & a_{99}, \ldots, & a_{3 n}, \ldots,
\end{array}
$$

which correspond to a sequence $\beta_{1}, \beta_{2}, \beta_{8}, \ldots$ of ordinal numbers of which $\gamma$ is the limiting number, by traversing the sequences diagonally, and thus $a_{11}, a_{22}, a_{89}, \ldots$ is the sequence which corresponds to $\gamma$.

Every sequence $b_{1}, b_{2}, b_{3}, \ldots$ is to be such that $b_{1}<b_{2}<b_{3}, \ldots$ It is pointed out by Hardy that an infinite freedom of choice arises in the case of each limiting number $\gamma$, since there are an indefinite number of sequences of the preceding ordinal numbers, of each of which sequences $\gamma$ is the limiting number. Thus, for example, $\omega^{2}$ is not only the limit of $\omega, \omega .2, \omega .3, \ldots$, but also of $\omega+1, \omega .2+2, \omega .3+3, \ldots$. In the case of the smaller limiting numbers of the second class. Hardy shews how to exercise this freedom of choice so as to obtain distinct sequences; thus $\omega$ is taken as the limit of $1,2,3, \ldots ; \omega^{2}$ is taken as the limit of $\omega, \omega .2, \omega .3, \ldots$; in particular, $1,5,17,49,129, \ldots$ is obtained as the sequence corresponding to $\omega^{2}$.

In order that the method should really suffice to define a sequence which shall correspond uniquely to each prescribed number $\beta$ of the first or of the second class, it would be necessary to replace the freedom of choice by a definite norm, or finite set of rules, which would decide in the case of any particular limiting number $\gamma$, of which particular sequence of the preceding ordinal numbers it must be regarded as the limit, for the purpose of forming the sequence of integers which is to correspond to $\gamma$, in accordance with the mode of formation prescribed in (2).

Hardy has given no norm of this character, but confines himself to the selection of the sequences which are to correspond to some of the lower limiting numbers of the second class; thus he finds

$$
\begin{array}{cc}
\left(\omega^{2} .2\right) & 1,17, \ldots, \\
\left(\omega^{3}\right) & 1,17, \ldots, \\
\left(\omega^{\omega}\right) & 1,5, \ldots, \\
\left(\epsilon_{1}\right) & 1,5, \ldots
\end{array}
$$

When we reach the region of the e-numbers of the second class, it is difficult, if not impossible, to imagine the nature of the norm which would 
suffice to make the decision referred to above, and no such norm is in fact forthcoming. On this ground, the method cannot be regarded as really defining a set of points corresponding to all the numbers of the first and second classes.

Moreover, it can be shown that, if sequences such as those contemplated by Hardy are employed, the second figure $b_{2}$ would have indefinitely great values for numbers $\beta$ of the second class, and thus that for sufficiently great ordinal numbers of the second class the corresponding sequences can have no existence.*

Corresponding to $\omega^{\omega}$, Hardy finds the sequence $1,5, p, q, \ldots(p>5)$. With the mode of defining the sequences, as in (2), corresponding to higher limiting numbers, for all numbers $>\omega^{\omega}$, the second figure in the sequences will in no case be $<5$, and, in fact, Hardy finds for the number $\epsilon_{1}$ defined as the limit of $\omega, \omega^{\omega}, \omega^{\omega}, \ldots$, a sequence $1,5, p^{\prime}, q^{\prime}, \ldots\left(p^{\prime}>5\right)$. For any number $>\epsilon_{1}$ the second figure will be not less than $p^{\prime}$; thus $\epsilon_{1}+1$ has $5, p^{\prime}, q^{\prime}, \ldots$ for its sequence, and for $\epsilon_{1}+\omega$ the second figure of the sequence is $p^{\prime}$. It follows that, with the mode of formation contemplated, the second figure of the sequence corresponding to $\epsilon_{2}$, defined as the limit of $\epsilon_{1}, \epsilon_{1}^{e_{1}}, \epsilon_{1}^{e_{1}}, \ldots, \ldots$ is not less than $p^{\prime}$. Proceeding in this way, we see that for the numbers $\epsilon_{1}, \epsilon_{2}, \epsilon_{9}, \ldots, \epsilon_{n}, \ldots$ the second figure of the sequence must continually increape as $n$ is increased. Now consider any sequence $k_{1}, k_{2}, \ldots, k_{n}, \ldots$ of in reasing integers; there is a number $\beta_{1}$, from and after which the $a_{2}$ is a ways $>k_{1}$; again, there is a number $\beta_{2}$, from and after which the $a_{2}$ is always $>k_{2}$, and so on. The number $\beta_{u}$, which is also a number of the second class, being the limiting number of the sequence $\beta_{1}, \beta_{2}, \beta_{3}, \ldots$, is such that in the sequence of integers which corresponds with it, the $a_{2}$ is greater than $k_{n}$ for every value of $n$, and is therefore indefinitely great. There can therefore exist no sequence which corresponds to $\beta_{\omega}$; hence it is impossible to construct in the ruanner contemplated an unenumerable set of points. The point here discussed was not considered by Hardy, probably simply because he did not attempt to set up a definite norm for the sequences.

W. H. Young has employed $†$ Bernstein's diagrammatic method, to correlate ordinal numbers of the second class with points of the continuum. The method does not, however, amount to the construction of a set of points of cardinal number $N_{1}$, in accordance with the vie $/$ maintained in

- Prof. W. Burnside communicated to me that he had convinced himself of the truth of this statement. I then devised the proof of its correctness given in the text.

+ Proc. London Math. Soc., Ser. 2, Vol. 1, p. 243. 
the present communication as to the necessity for a definite norm which shall determine the correspondence.

13. In case the criticism which has been here given of the general theory of order types and of aleph-numbers be accepted as wholly, or in part, valid, the debt which mathematical science owes to the genius of G. Cantor will be in no respect diminished. The fundamental distinction between enumerable and unenumerable aggregates, the ordinal theory of the continuum, the theory of irrational numbers, with the interpretation of the arithmetical theory of limits, and the conception of the transfinite ordinal numbers of the second class, with their applications to the theory of sets of points, remain as permanent acquisitions which rest upon a firm logical basis. This order of ideas has already become indispensable for purposes of formulation in analysis and in geometry; it is constantly receiving new applications, orving to its admirable power in providing the language requisite for expressing results in the theory of functions with the highest degree of rigour and generality. Cantor's creations have rendered inestimable service in formulating the limitations to which many results in analysis, formerly supposed to be universally valid, are subject.

The outlying parts of the theory to which exception has been taken in the present communication, do not appear to be comparable in inportance, for the general purposes of analysis, with those parts to which the criticisms made are inapplicable. The latter involve only a natural extension of the notion of numbers, in which account is taken of all the elements which are essential in the conception of number in its original form; whereas an endeavour has here been made to shew that the more speculative general theory of aleph-numbers and order types depends upon an extension of the notion of numbers which leaves out of account an essential element in that conception, viz., the notion of correspondence ; and that this is the origin of the contradiction which ensues when an endeavour is made to contemplate the totality of these new entities.

The criticisms here given are advanced with some diffidence, on account of the great logical difficulties of the subject, and especially of the philosophical difficulties involved in questions relating to existential propositions. It is hoped, however, that the remarks made in this communication may, in any case, be of some utility as a contribution towards the discussion of questions of great interest which, at the present time, cannot be regarded as having been decisively settled. 